\title{
Les intendants de la Nouvelle-France et la République des Lettres
}

\section{Jean-Claude Dubé}

Volume 29, numéro 1, juin 1975

URI : https://id.erudit.org/iderudit/303416ar

DOI : https://doi.org/10.7202/303416ar

Aller au sommaire du numéro

Éditeur(s)

Institut d'histoire de l'Amérique française

ISSN

0035-2357 (imprimé)

1492-1383 (numérique)

Découvrir la revue

Citer cet article

Dubé, J.-C. (1975). Les intendants de la Nouvelle-France et la République des Lettres. Revue d'histoire de l'Amérique française, 29(1), 31-48.

https://doi.org/10.7202/303416ar d'utilisation que vous pouvez consulter en ligne.

https://apropos.erudit.org/fr/usagers/politique-dutilisation/ 


\title{
LES INTENDANTS DE LA NOUVELLE-FRANCE ET LA RÉPUBLIQUE DES LETTRES
}

\author{
Jean-Claude Dubé \\ Département d'histoire \\ Université d'Ottawa
}

La Gazette de Hollande du 11 novembre 1738 commentait de la façon suivante le décès de Claude-Thomas Dupuy, ancien intendant de Québec: "Il avait une infinité de projets de mécanique auquel il aurait mis la dernière main à son retour de Bretagne. La République des Lettres a fait en lui une grande perte ${ }^{1}$."

La République des Lettres, c'est l'ensemble du monde des savants et lettrés européens des $17^{\mathrm{e}}$ et $18^{\mathrm{e}}$ siècles qui, par les salons, les sociétés de pensée, les correspondances et les revues communiquent entre eux indépendamment des frontières politiques; la conscience qu'ils ont de constituer, par leur science et leurs qualités intellectuelles, un monde à part les amène à se considérer comme les citoyens d'un Etat consacré uniquement aux choses de l'esprit 2. Claude-Thomas Dupuy, par ses recherches en physique et par ses inventions avait attiré l'attention des savants ; l'Académie des Sciences avait parlé de lui, les revues de l'époque avaient fait état de ses expériences ${ }^{3}$. Il était membre de cette nation intellectuelle.

Il y a lieu de se demander si cet homme fait figure d'exception parmi les magistrats qui furent envoyés de Paris à l'intendance de Québec, ou si plutôt les commissaires royaux chargés d'administrer le Canada étaient des hommes non seulement compétents pour leur tâche, mais aussi cultivés ou même savants.

Notre intention est donc d'aborder ici une question sur laquelle on a très peu écrit: le niveau intellectuel des administrateurs que le gouvernement français destinait au Canada. Nous voudrions mesurer quelles étaient l'étendue et la profondeur de

1 J.-C. Dubé, Claude-Thomas Dupuy, intendant de la Nouvelle-France (Montréal, 1969), 347.

2 A. Furetière, Dictionnaire universel ... (Paris, 1691) : “on dit aussi la République des Lettres, en parlant collectivement de tous les gens d'étude".

3 J.-C. Dubé, op. cit. 339 ss.

RHAF, vol. 29, no 1 (juin 1975) 
leur culture, savoir s'ils participaient à la vie scientifique de leur temps. Les recherches que nous poursuivons sur l'origine sociale des intendants nous permettent d'apporter à ces questions quelques éléments de réponse.

Les sources que nous utilisons, ce sont avant tout les minutes notariales; elles nous ont permis, encore plus que les registres paroissiaux, de reconstituer les familles (parfois sur plus de 5 générations) et de suivre l'évolution des professions. Les inventaires après décès surtout nous ont fourni de précieux renseignements sur le mode de vie; ils sont d'ordinaire bien faits: description détaillée, indications de la prisée. Nous n'avons pas eu la chance malheureusement de retrouver le notaire de tous les intendants; il nous manque ainsi cinq inventaires, peut-être sont-ils d'ailleurs inexistants ou ont-ils disparu. Nous n'avons pas retrouvé celui de Bouteroue (ni celui de son père) ; il en va de même pour Duchesneau, pour Bochart (celui de son père a brûlé durant la Commune), pour Chazel et pour Bigot (nous avons l'inventaire du père de ce dernier). Mais même pour ceux-là nous avons d'autres contrats notariaux et ne sommes donc pas complètement dépourvu, au moins à certains points de vue. Nous avons aussi puisé dans quelques autres séries des Archives Nationales, comme les fonds coloniaux (série E surtout) pour Dupuy. Les séries manuscrites de la Bibliothèque Nationale ont aussi fourni des documents concernant Beauharnois et Bouteroue.

Nous passerons en revue les quinze titulaires nommés au poste de Québec entre 1663 et 1760 . Trois de ceux-là ne mirent pas les pieds dans la vieille capitale: Louis Robert de la Fortelle, nommé en 1663, mais qui resta en Europe; Edme-Nicolas Robert, mort en mer en 1724; Guillaume de Chazel, naufragé en 1725 ; ils sont peu connus. Les douze autres sont moins inconnus.

Nous essaierons de saisir le milieu familial de ces personnages et de voir s'il leur procurait une ambiance favorable à l'épanouissement de leurs facultés intellectuelles. Nous dirons ensuite dans quel moule ils ont été formés: par quel collège ou faculté ils sont passés. Nous passerons enfin à leurs performances propres, c'est-à-dire à ce que nous savons de leurs activités dans le monde scientifique; ou nous tâcherons de tirer parti des renseignements que nous donnent les catalogues de leurs bibliothèques ou de déceler leurs goûts, tels que nous les révèlent les inventaires de leurs biens.

Il a été possible de retrouver la famille immédiate de tous les intendants, et souvent même de remonter assez loin dans le temps; 
nous avons pu recueillir sur l'une ou l'autre d'importantes précisions sur les points qui nous intéressent. Une exception cependant: la famille de Jacques III Duchesneau nous échappe presque totalement: il se peut que Jacques II, son père, ait été avocat et ait donc fait des études dans une Faculté de droit, car la charge de trésorier de France, dont il était revêtu ${ }^{4}$, était généralement accordée à des gradués en droit ${ }^{5}$.

Le milieu familial le plus remarquable est sans doute celui de Michel VI Bégon ${ }^{6}$. Son oncle, Scipion, était docteur en théologie de la Sorbonne; son frère cadet, Jérôme-Scipion, l'était aussi et fit une très brillante carrière dans l'épiscopat. Une de ses sœurs entra, par son mariage, dans une famille où la culture était à l'honneur, les Barrin de la Galissonière ?. Son père, Michel V, étudia chez les Jésuites et fit son droit à Paris avant d'entrer dans la magistrature locale puis de passer au service de la marine où il s'illustra. Il s'était acquis une solide réputation dans les milieux érudits; il avait de nombreux correspondants. Il est l'exemple typique du collectionneur averti, aux intérêts diversifiés. Il augmenta considérablement la bibliothèque que lui avait léguée son père, "homme fort cultivé", selon Y. Bézard ${ }^{8}$, signe qu'une tradition d'intérêt aux choses de l'esprit le précédait. Ses riches collections couvraient des domaines variés: numismatique, gravure, peinture, ethnographie, histoire naturelle. C'est d'ailleurs en son honneur qu'une plante américaine, devenue ensuite très populaire, a été appelée bégonia; et c'est grâce à son trésor d'estampes que le livre de Charles Perrault, Les hommes illustres, put être réalisé.

Le premier intendant nommé pour le Canada, Louis Robert de Fortelle, qui ne mit jamais les pieds en Amérique, passa près de vingt ans dans les intendances militaires, avant d'acheter une charge de président à la chambre des comptes. Il était le rejeton d'une grande famille de juristes, dont deux au moins furent régents à l'Université d'Orléans au XVIe siècle ${ }^{9}$. La famille se

4 Bibliothèque Nationale, Paris (ci-après BN), Section des Manuscrits, (Mss), Cabinet d'Hozier, 92.

5 J.-P. Charmeil, Les trésoriers de France à l'époque de la Fronde (Paris, 1964), 24.

6 Y. Bézard, Fonctionnaires maritimes et coloniaux sous Louis XIV. Les Bégon (Paris, 1932).

7 Dictionnaire de biographie française (DBF), Jacques I Barrin, mort en 1645, fut maître des requêtes et conseiller d'Etat; Jacques II, beau-père de Catherine Bégon, fut aussi maître des requêtes et intendant; il avait la réputation d'être un "bel esprit, savant et un peu intéressé".

8 Y. Bézard, op. cit., 14-15.

$9 \mathrm{~F}$. Bluche, L'origine des magistrats du Parlement de Paris au XVIIIe siècle (1715-1771). Dictionnaire généalogique (Paris, 1956), 370s. 
transporta ensuite à Paris où le grand-père de Louis, Anne Robert, connut la renommée. Ses plaidoyers furent imprimés en latin et en français et plusieurs fois réédités ${ }^{10}$; certains furent même publiés conjointement avec ceux d'Antoine Arnauld ${ }^{11}$, le père des célèbres jansénisites, décédé deux ans après lui. Nous avons eu la chance de retrouver la bibliothèque de ce fameux avocat ${ }^{12}$, une bibliothèque remarquable ${ }^{13}$ pour l'époque: 1451 volumes, dont plus de 400 in-folio (dont 3 Plantin). Des 391 titres identifiables, 140 sont des ouvrages de droit: $36 \%$; les lettres anciennes, 112: 29\%; les lettres modernes, 21: $5 \%$; la théologie (Ecriture sainte et Pères de l'Eglise surtout), 57: $15 \%$; l'histoire et la géographie, 51: 13\%. La forte proportion d'ouvrages de jurisprudence confirme la grande réputation de notre homme (la moyenne chez les magistrats parisiens du XVIIIe siècle se situe autour de $18 \%$ ) $^{14}$.

Sur les enfants d'Anne Robert nous savons peu de choses: deux filles entrèrent au couvent, une autre épousa un avocat au Grand Conseil, Antoine du Laurens, et une dernière se maria dans une famille en pleine ascension sociale, les Chauvelin, qui étaient alliés aux Le Tellier. Les deux fils étudièrent probablement le droit, puisque Nicolas, père de Louis, devint trésorier de France, et Julien, conseiller au Parlement. Deux des frères de Louis obtinrent leur doctorat en théologie ${ }^{15}$. Il s'agit donc d'une famille où les traditions d'études étaient florissantes depuis de très nombreuses années.

Nous ne retrouvons pas dans les autres familles des célébrités comparables aux deux premières, mais elles présentent pour la plupart des aspects très intéressants, et leur manque d'éclat les rend d'autant plus représentatives de la moyenne de leur milieu. Prenons les Bochart, par exemple. De Jean IV à Jean VII, père de Jean VIII, intendant de la Nouvelle-France, on est maître des requêtes, puis intendant de façon continue; à 1596).

10 Anne Robert, Annaei Roberti, Rerum judicatarum libri IV (Paris,

11 Louis Servin, Actions notables et plaidoyez de Messire Loys Servin, avec les plaidoyers de M. A. Robert, Arnault et autres (Rouen, 1629).

12 Archives Nationales de France (ci-après AN), Minutier Central (MC), étude XVIII : liasse 218, 14 juillet 1617, inventaire.

13 H.-J. Martin, Livre, pouvoirs et société à Paris au XVIIe siècle (1598-1701) (Genève, 1969), 492. L'A. a aralysé 200 inventaires pour préparer son tableau des bibliothèques "moyennes et petites" pour la période 1601-1641; deux seulement ont plus de 1000 titres.

14 F. Bluche, Les magistrats du Parlement de Paris au XVIIIe siècle (1715-1771) (Paris, 1960), 291.

15 AN, MC, CXIX: 26, 21 juin 1677. Inventaire. 
une seule exception près, Jean VI, mais il est conseiller d'Etat. Il était pratiquement nécessaire, pour de telles carrières, de passer par la Faculté de droit. Trois des frères de Jean VIII étudient la théologie et entrent dans les ordres; Guillaume, docteur en Sorbonne, devient archidiacre de l'Eglise de Rouen, puis évêque de Valence ${ }^{16}$.

Le père d'Antoine-Denis Raudot, Jacques, intendant de Québec conjointement avec lui, avait obtenu baccalauréat et licence de la Faculté de droit ${ }^{17}$; deux de ses tantes s'étaient alliées à des familles qui avaient connu un certain renom dans les cercles littéraires français, les Vauquelin et les Carcavy ${ }^{18}$.

Le père de François Bigot, Louis-Amable, avait aussi fait des études, puisqu'il commença sa carrière comme avocat au Parlement de Bordeaux. Il en fut vraisemblablement de même pour Joseph, frère aîné de François, qui devint conseiller au même parlement ${ }^{19}$.

Claude II Bouteroue, père du remplaçant temporaire de Talon, était procureur au Parlement et donc gradué en droit. La famille Bouteroue était originaire de Meaux où Claude I et Jean exerçaient le notariat ${ }^{20}$. Claude III entra dans une famille d'orfèvres très connus de Paris ${ }^{21}$.

Les Beauharnois formaient, comme les Robert, une des familles les plus en vue d'Orléans ${ }^{22}$. Observons les ascendants directs de François V, qui vint au Canada en 1702. Son père, François IV, fut avocat au Parlement avant de devenir directeur général des finances et gabelles de la Généralité d'Orléans. Son grand-père, Jean II, était "licencié ès droits de l'Université d'Orléans" et son arrière-grand-père, François II, fit une intéressante carrière dans la magistrature locale avant d'être nommé conseiller d'Etat. Voilà encore une tradition au moins séculaire d'études juridiques. Notons aussi que François IV avait comme cousin germain un grand érudit, Nicolas Thoynard ${ }^{23}$, qui fut

${ }_{16}$ R. Mousnier, Lettres et mémoires adressés au Chancelier Séguier (Paris, 1964), 1186ss.

$17 \mathrm{BN}$, Mss, Pièces originales, 2437.

18 BN, Mss, Dossiers bleus, 557.

19 Archives Départementales (ci-après AD) de la Gironde, $3 \mathrm{E} 17581$, 14 mars 1766, mariage d'Anne de Bigot.

$20 \mathrm{AN}$, MC, XXI: 160. 15 décembre 1651, Partage Bouteroue. AD, Seine-et-Marne, Minutes du notaire Jean Bouteroue, 1553-1571.

21 AN, MC, LXVI: 99, 25 juin 1644, mariage Bouteroue-Lescot.

22 BN, Mss, Pièces originales, 242.

${ }^{23}$ L. Moreri, Le grand Dictionnaire historique (Paris, 1759). 
admis dans le cercle des frères Dupuy et s'intéressa entre autres à l'Ecriture sainte et à la chronologie, et que la sœur de François V, Elisabeth, entra dans la famille Bégon, en épousant Michel VI.

Quant à la famille de Henri de Chazel, qui venait prendre la relève de Bégon en 1725, quand il périt dans le naufrage du Chameau, elle s'était taillé une place de choix dans la société nîmoise. Le père de l'intendant, qui avait été reçu docteur en droit en 1658, fit une brillante carrière dans la magistrature et fut l'un des membres fondateurs de l'Académie de Nîmes ${ }^{24}$. Il avait épousé Françoise de Cassagne, dont le père, Michel, et les oncles étaient magistrats à Nîmes; l'un d'eux, Jacques, était même un des correspondants de Peiresc, le grand humaniste du Midi. Deux des frères de Françoise doivent aussi retenir notre attention: Jacques, qui fut docteur en théologie, accéda à l'Académie française et participa à la création de l'Académie des Inscriptions ${ }^{25}$; l'autre, Henri, contribua comme Jean-Pierre de Chazel, à la fondation de l'Académie de Nîmes ${ }^{26}$.

Pour trois autres des intendants de la Nouvelle-France nous savons que de leurs proches parents avaient étudié la théologie. L'oncle de Gilles Hocquart, Claude, était bachelier; un de ses frères, Jacques-François, était "prêtre chanoine de l'Eglise cathédrale du Mans" ${ }^{27}$. L'inventaire de son père, Jean-Hyacinthe, contient des articles qui nous ont paru intéressants ${ }^{28}$ : "globes terrestre et céleste", nombreuses cartes géographiques, estampes et miniatures, " 186 volumes de livres ... traitant de différents sujets d'histoire et de dévotion", "deux urnes de pierre antique", "plusieurs objets en porcelaine fine...", "un clavecin de bois de noyer". Edme-Nicolas Robert avait un oncle chanoine de l'Eglise royale et collégiale de Champeaux. Ses deux sœurs s'étaient alliées à d'importantes familles de robe, les Leclerc de Lesseville ${ }^{29}$ et les Du Maitz de Goimpy ${ }^{30}$. Quant à Talon, trois de ses frères, plus âgés que lui, firent carrière dans le clergé ${ }^{31}$ : Antoine, bachelier en théologie et sous-chantre en l'Eglise cathédrale de Châlons, qui résignait son poste, en 1661, en faveur de son jeune frère, Arthus Talon, déjà chanoine de l'Eglise Notre-

24 Notes de M. Joffre, archiviste des AD du Gard.

$25 \mathrm{DBF}$ et Mémoires de l'Académie de Nîmes, IX (1886) : 248.

26 Notes de M. Joffre, voir note 24.

27 AN, MC, LXXVII: 192, 24 mai 1726, compte Michelet du CosnierHocquart.

28 Ibid.: 183, 7 fév. 1724 .

29 Ibid.: LVIII, 191, 29 avril 1696, mariage Leclerc de Lesseville-Robert.

30 Ibid.: 228, 6 mars 1707, mariage Du Maitz-Robert.

31 BN, Mss, Chérin, 192. 
Dame de Reims et curé de Bligny; Nicolas, qui fut chanoine et sous-chantre de l'Eglise de Notre-Dame de Vaux et abbé de Toussaints, abbaye située à Châlons.

Il nous reste enfin deux personnages au sujet desquels les indications sont plus minces, mais pas nécessairement moins significatives, si l'on en juge par leurs propres performances. La mère de l'intendant De Meulles était de la célèbre famille Briçonnet ${ }^{32}$ qu'avait illustrée entre autres ce cardinal qui devint un des chefs de la pré-réforme française. Sa sœur, Elisabeth, épousa un conseiller au Parlement et lui-même devint, par son mariage, le beau-frère de Michel V Bégon, dont nous avons retracé la remarquable activité intellectuelle. ${ }^{33}$ Claude Dupuy ${ }^{34}$, père de l'intendant, était marchand-papetier à Paris; l'on peut supposer que ses nombreux contacts avec les libraires parisiens l'ont enrichi intellectuellement et que son fils y prit le goût des livres. Deux des frères de Claude, Georges et Jean, se firent prêtres. Si nous nous tournons du côté de la mère de l'intendant, Elisabeth Aubry, nous constatons qu'un de ses frères, Jacques, détenait un doctorat en théologie et que l'autre, Charles, étudia le droit et acheta la charge d" "avocat es conseils de Sa Majesté". Le fils de ce dernier, Jacques-Charles, donc le cousin germain de Claude-Thomas Dupuy, fut l'un des avocats les plus renommés de la capitale au début du XVIIIe siècle.

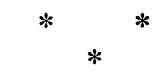

Si le milieu familial des intendants de la Nouvelle-France est déjà aussi riche intellectuellement que nous avons pu le constater, il y a tout lieu de supposer que leur éducation a été soignée; nous pourrions déjà le déduire de la simple lecture de leur correspondance; s'ils ne sont pas tous des stylistes, leur langue est claire et correcte et contient souvent des réminiscences de leurs études classiques.

Mais nous avons sur certains d'entre eux des précisions concernant le cours d'études qu'ils ont suivi. Nous savons de façon certaine que cinq étudièrent le droit. Bouteroue était avocat aux Requêtes du Palais en 1634, c'est-à-dire dès l'âge de $18 \mathrm{ans}^{35}$. Jacques Raudot passait en janvier 1666 son baccalauréat et sa licence à Paris ${ }^{36}$. Michel Bégon obtenait sa licence à Orléans en

32 AN, MC, CXIII: 26, 29 oct. 1650, inventaire Elisabeth Briçonnet.

33 Ibid., XCII : 211,13 août 1675 , renonciation.

34 J.-C. Dubé, op . cit., passim.

35 AN, Z1B : 567.

36 BN, Mss, Pièces originales, 2437. 
janvier $1694^{36 a}$. Edme-Nicolas Robert entrait au Parlement de Paris en 1696, comme conseiller du roi substitut du procureur général ${ }^{37}$. Quant à Claude-Thomas Dupuy nous avons retrouvé la mention de chacune des étapes de son cours de droit, baccalauréat en 1697, licence l'année suivante; il s'inscrivit même aux cours de doctorat, avant de devenir avocat au Parlement de Paris ${ }^{38}$.

Les archives des facultés de droit étant fort lacunaires pour l'Ancien Régime, nous ne pouvons nous assurer que les autres intendants y aient passé. Mais il est plausible que Jean Bochart, Louis Robert et Antoine-Denis Raudot aient suivi les traces de leur père. Pour les sept autres nous n'avons encore trouvé aucune indication.

Tous les étudiants en droit avaient d'abord fréquenté un collège. Claude-Thomas Dupuy passia six ans au Collège de Presles-Beauvais, qui se trouvait situé tout près du domicile de son père; le minutier central de Paris conserve même l'affiche qu'il publia à l'été de 1696 pour annoncer la soutenance publique de ses "thèses", exercice qui couronnait ordinairement la fin d'un cours collégial ${ }^{39}$. Michel Bégon, pour sa part, resta en France, alors que ses parents étaient partis à la Martinique (de 1682 à 1685), pour terminer ses études ${ }^{40}$. Il n'est pas impossible qu'il ait étudié au Collège Louis-le-Grand (appelé auparavant Collège de Clermont) où son frère cadet, Jérôme-Scipion, remporta de nombreux prix en 1697. Remarquons aussi que son père le fit entrer, tout de suite après ses études, dans l'administration maritime, en lui obtenant un brevet d'écrivain principal ${ }^{41}$. C'est la formule que nous retrouvons pour d'autres intendants au XVIIIe siècle; Bigot par exemple commença lui aussi comme écrivain dans un port de mer. ${ }^{42}$

Talon avait fréquenté le Collège de Clermont, célèbre institution dirigée par les Jésuites.

La première éducation que j'ai reçue par leurs soins obligeans, écrivait-il au P. Oliva en 1666, m'ayant élevé dans les écoles, demande toute ma reconnaissance. $\mathrm{Si}$ j'avais conservé le fruit

36a Y. Bézard, op. cit., 19.

37 BN, Mss, Chérin, 173.

38 J.-C. Dubé, op. cit., 48.

39 Ibid., 43ss.

40 Y. Bézard, op. cit., 268.

41 Ibid.

42 G. Frégault, François Bigot, administrateur français (Montréal, 1948), I: 67 . 
de leurs instructions, j'aurais l'honneur de vous en faire part par un discours latin. (Le P. Oliva lui avait écrit en latin). Mais je suis devenu meschant escolier de bon maistre ... ${ }^{43}$

Il avait peut-être perdu l'usage du latin, mais pas le goût des choses de l'esprit; ne le vit-on pas participer activement, et avec le plus grand plaisir, semble-t-il, à une dispute publique au Collège de ces mêmes Jésuites en 1666.

Les premières disputes de philosophie se font dans la Congregation avec succez. Toutes les puissances s'y trouvent, $\mathrm{Mr}$ l'intendant entr'autres y a argumenté très bien. Mons. Joliet et Pierre Francheville y ont très bien repondu a toute la logique ${ }^{44}$.

Milieu familial stimulant, éducation soignée (on peut prudemment le supposer pour la plupart d'entre eux) donnèrent aux intendants de la Nouvelle-France une solide formation classique et même professionnelle. Pour certains ce fut en même temps le point de départ d'une carrière "scientifique". Il faut les classer parmi les savants de leur temps.

Claude de Bouteroue était numismate et il publia en 1666 chez Sébastien Cramoisy un gros in-folio - pour lequel il reçut une gratification de Colbert - sur les monnaies de France: Recherches curieuses de monnoyes de France depuis le commencement de la Monarchie. Son livre ne traitait cependant que de l'époque mérovingienne. Ses notes manuscrites, qui couvraient une bien plus large période, furent reprises par François Leblanc, qui publia en 1690 un Traité historique des monnoies de France ${ }^{45}$. $J$ 'ai retrouvé à la Bibliothèque Nationale ${ }^{45 a}$ des épaves de sa correspondance avec quelques-uns de ses amis du monde scientifique: le Président Lamoignon, dont l'influence était grande dans les milieux savants de la capitale ${ }^{46}$, et Samuel Bochart. Ce dernier, brillant controversiste protestant, publia plusieurs ouvrages sur l'Ecriture sainte et fut invité à Stockholm par Christine de Suède ${ }^{47}$.

Claude-Thomas Dupuy, pour sa part, s'intéressait aux sciences naturelles, et tout spécialement à la physique ${ }^{48}$. Il consacra

43 C. de Rochemonteix, Les Jésuites et la Nouvelle-France au XVIIe siècle (Paris, 1895-6), III : 85. 345.

${ }^{44}$ Le Journal des Jésuites (éd. Laverdière et Casgrain, Québec, 1871),

45 DBF.

45a BN, Mss Frs, n.a., 2488-2489.

46 H.-J. Martin, op. cit., 924.

$47 \mathrm{DBF}$.

48 J.-C. Dubé, op. cit., 339ss. 
les loisirs que lui laissait sa retraite anticipée à construire des machines à élever l'eau. Ses inventions furent mises à l'épreuve près de Paris et dans les mines bretonnes. L'Académie des sciences suivit ses recherches avec attention; les revues "scientifiques" du temps en parlèrent assez longuement. Moreri lui trouve des talents dans les "sciences et les beaux-arts" et lui décerne même, mais faussement, semble-t-il, la paternité des sphères mobiles fabriquées pour illustrer le système copernicien. Il avait apporté avec lui à Québec son cabinet de physique. Il aida même Sarrazin dans quelques dissections.

Antoine-Denis Raudot fit très rapidement impression, lui aussi, dans la colonie par ses hautes qualités intellectuelles et l'équilibre de sa personnalité. Les volumineux rapports qu'il a laissés sur l'économie canadienne et sur l'ethnographie sont clairs et lucides ${ }^{49}$. M. Marcel Giraud a indiqué, dans son Histoire de la Louisiane, le rôle de premier plan que Raudot joua à Versailles, avant et après sa nomination à la direction de la Compagnie des Indes, dans le groupe d'hommes éclairés qui menaient le ministère de la Marine. La géographie, les sciences naturelles, l'ethnologie l'intéressaient passionnément, et il mit à leur service le crédit qu'il avait à la cour ${ }^{50}$. Il fréquenta assidûment le cercle qui se réunissait autour du Maréchal d'Estrées. Celui-ci, qui partagea avec Raudot la direction de la Compagnie des Indes, était une des figures dominantes du monde scientifique de la Capitale; il remplaça Vauban à l'Académie des sciences en 1707; il fut ensuite élu à l'Académie française, puis à l'Académie des Inscriptions ${ }^{51}$.

Nous avons aussi trouvé à la Bibliothèque Nationale une intéressante correspondance entre François de Beauharnois et le célèbre bénédictin de Saint-Germain-des-Prés ${ }^{52}$, Dom Montfaucon. Celui-ci inséra dans le 4e tome des Monuments de la Monarchie une dissertation que l'intendant de Rochefort (poste qu'il occupa quelques années après son passage à Québec) avait préparée sur un tombeau ancien découvert dans l'Ile de Ré.

Il nous est donc possible d'affirmer que quelques-uns au moins des intendants de la Nouvelle-France participèrent activement à la vie scientifique de leur époque; quatre sur quinze, cela nous a paru une proportion fort impressionnante - on ne peut

49 Dictionnaire biographique du Canada (DBC).

50 M. Giraud, Histoire de la Louisiane française (Paris, 1958), II: 16.

$51 \mathrm{DBF}$.

$52 \mathrm{BN}$, Fonds français, 17702. 
en dire autant des gouverneurs, par exemple, qui, à l'exception de La Galissonière, ne représentèrent rien dans la République des Lettres. Pas de surprise dans cette constatation, puisque l'élément intellectuel dynamique du Royaume se retrouvait majoritairement dans la bourgeoisie et tout spécialement dans la magistrature.

Mais si les autres intendants ont des performances moins spectaculaires, l'étude de leurs dossiers n'en est pas moins instructive: elle nous met en présence de personnages qui se situent plus près de la moyenne de leur groupe social, comme nous l'avons indiqué plus haut. C'est pourquoi il nous faut maintenant essayer de tirer parti des indications que nous donnent les inventaires après décès. Deux éléments retiendront ici notre attention, la liste des livres et le mobilier.

Il y a d'utiles renseignements à tirer de l'importance numérique et de la composition de leurs bibliothèques. Les dix inventaires que nous possédons mentionnent des livres, mais l'énumération n'est détaillée que dans cinq cas; il s'agit d'ordinaire pour les autres d'une description en vrac: le nombre des livres et la prisée. Le cas de Talon fait problème: on ne trouve dans son appartement de la rue du Bac qu'un "Atlas en douze volumes in folio relié en parchemin avec les cartes", prisé trente livres ${ }^{53}$. Avait-il fait don de sa bibliothèque ? L'avait-il vendue? Nous n'avons pas encore de réponse à ces questions.

La plus importante est celle d'Antoine-Denis Raudot ${ }^{54}$ "environ 2,000 volumes de livres tant in folio in quarto in douze et in seize traitant de differents sujets et histoire", prisée 1,500 livres. Celle de Dupuy vient en second lieu, et bien loin derrière, avec ses 1,200 volumes ${ }^{55}$. Edme-Nicolas Robert en a pour sa part $763{ }^{56}$, Hocquart ${ }^{57}, 545$, Louis Robert 58,414 , Bégon ${ }^{59}, 230$, Beauharnois ${ }^{60}, 205$, De Meulles ${ }^{61}, 140$ et Jacques Raudot ${ }^{62}$, 83.

Pour celles dont la description est détaillée, au moins partiellement, il y a moyen de pousser l'analyse en nous servant du

53 Rapport des Archives du Québec, 46 (1966) : 61.

$54 \mathrm{AD}$, Seine et Oise (Versailles), 2 août 1737.

55 J.-C. Dubé, op. cit., 317ss.

56 AN, MC, LVIII: 280,29 janvier 1725 , Inventaire.

57 AN, MC, II : 711,24 avril 1783, Inventaire.

58 AN, MC, CXIII: 220,26 juillet 1706 , Inventaire.

$59 \mathrm{AD}$, Loir et Cher, étude 19, 559, 2 mai 1747, Inventaire.

$60 \mathrm{AD}$, Loiret, 3E 10523, Inventaire.

61 AN, MC, LXXVII: 120, 26 avril 1711, Inventaire déposé.

62 AN, MC, LXVII : 438, 5 mars 1728, Inventaire. 
tableau préparé par François Bluche dans son étude sur les magistrats parisiens ${ }^{63}$. La classification usuelle au XVIIIe siècle pour les livres est celle qu'on retrouve dans la liste dressée par Dupuy à son retour à Paris: la théologie (c'est-à-dire tout ce qui a trait à l'Ecriture sainte et à la religion), la jurisprudence, les sciences et les arts, les Belles-lettres (modernes et anciennes), l'histoire (incluant les livres de géographie et les sciences dites auxiliaires de l'histoire). Nous avons fait le compte des titres identifiables et établi des pourcentages que nous présentons sur un tableau. Pour fin de comparaison nous avons ajouté les taux que Bluche a publiés dans son livre pour les bibliothèques des magistrats, dont les inventaires ont été faits entre 1734 et 1760 , puis entre 1766 et 1780 .

Le tableau met en évidence certains faits intéressants. EdmeNicolas Robert a une section de jurisprudence qui est très développée, plus même que la moyenne des magistrats parisiens, et qui, en chiffres absolus, dépasse celle de Dupuy, 112 ouvrages contre 105; donc un juriste, peu intéressé d'ailleurs dans les sciences et les arts (le taux le plus faible de tous les intendants). Quant à Dupuy, c'est précisément dans cette section qu'il excelle à cause de son intérêt pour les sciences physiques et pour la musique: $27.6 \%$, c'est pratiquement le double de ce qu'on retrouve chez les magistrats parisiens. Celle de Michel Bégon est assez spéciale: à peu près pas de livres de droit, ce qui est surprenant; mais pour la moitié ou presque, des livres de piété ou de théologie; par contre il a plusieurs numéros du Mercure galant et il possède le Bayle. Tous d'ailleurs ont celui-ci sur leurs rayons, sauf Hocquart. Beauharnois a de nombreux livres d'histoire et d'architecture. Hocquart, plusieurs livres de géographie et de nombreuses cartes; il semble s'intéresser à l'histoire d'Angleterre, possédant, entre autres, 23 volumes des débats de la Chambre des Communes.

Comme chez les magistrats parisiens encore, on retrouve sur leurs rayons des œuvres jansénistes, sauf chez Michel Bégon: la Bible de Sacy, les œuvres de Nicole (Hocquart, qui meurt dans le dernier quart du siècle, possède les œuvres de ce dernier, en 23 volumes), Carré de Montgeron; quelqu'un possède même les "Poésies de M. Arnauld". Témoignage de l'intérêt durable suscité par le jansénisme dans la robe. Tous ont des livres en langue étrangère: anglais, espagnol ou italien.

63 F. Bluche. Les magistrats du Parlement de Paris au XVIIIe siècle (1715-17r1) (Paris, 1960), 291. 


\begin{tabular}{|c|c|c|c|c|c|c|c|}
\hline & & $\begin{array}{c}\text { (m. 1725) } \\
\text { E. N. } \\
\text { Robert }\end{array}$ & $\begin{array}{c}\text { (m. 1738) } \\
\text { C.T. } \\
\text { Dupuy }\end{array}$ & $\begin{array}{c}\text { (m. 1747) } \\
\text { M. } \\
\text { Bégon }\end{array}$ & $\begin{array}{c}\text { (m. 1747) } \\
\text { F. de } \\
\text { Beau- } \\
\text { harnois }\end{array}$ & $\begin{array}{l}\text { (m. 1783) } \\
\text { G. } \\
\text { Hocquart }\end{array}$ & $\underset{\text { parisiens }}{\text { Magistrats }}$ \\
\hline \multicolumn{2}{|c|}{ Nombre de volumes } & 763 & 1200 & 230 & 205 & 549 & \\
\hline \multicolumn{2}{|c|}{$\begin{array}{l}\text { Nombre d'ouvrages } \\
\text { identifiables }\end{array}$} & 354 & 575 & 58 & 88 & 90 & $\begin{array}{l}1734-65 \\
1766-80\end{array}$ \\
\hline Théologie & $\%$ & 18.6 & 7.8 & 48 & 11.4 & 12.9 & $18.7 / 12$ \\
\hline Jurisprudence & $\%$ & 31.6 & 17.8 & 1.7 & 10.2 & 15.6 & $20.1 / 22$ \\
\hline Sciences et arts & $\%$ & 2.8 & 25.6 & 8.6 & 22.7 & 13.5 & $13.5 / 14$ \\
\hline Belles-lettres & $\%$ & 27.6 & 25.7 & 24 & 11.4 & 19 & $19 / 20$ \\
\hline Histoire & $\%$ & 19 & 22.8 & 17.2 & 44.3 & 39 & $27.5 / 32$ \\
\hline
\end{tabular}

Il reste enfin, pour compléter le portrait intellectuel des intendants de la Nouvelle-France à parcourir leurs inventaires pour y découvrir des indications sur leurs goûts, principalement leurs goûts artistiques. Ces documents comprennent toujours en effet un état détaillé du mobilier - ces "meubles meublants", qui forment, suivant l'expression d'un livre récent, le "décor de la vie"; tableaux, tapisseries, glaces, meubles, porcelaines, tables de jeu, instruments de musique, voilà quelques-uns des articles que nous retrouvons dans ces intérieurs.

Et d'abord ils possèdent tous des tableaux; certains même plusieurs. Ainsi Antoine-Denis Raudot, qui en a 115 (prisés 1,500 livres) ; De Meulles, 129, distribués dans les salles de son château de La Source; Talon, 98. Il s'agit dans l'ensemble de tableaux de peu de valeur, semble-t-il; signalons toutefois chez Raudot "un grand tableau du Bassan représentant divers sujets" (prisé 150 livres); on peut voir aussi à la Source une Magdelaine valant 200 livres; et, " 4 tableaux de fruits et un de poisson de Terburg" (prisé 350 livres), chez Louis Robert, un ensemble de 3 tableaux évalués à 500 livres. 
La mode des tableaux de famille n'est pas universelle. Quatre mentions seulement. François de Beauharnois en a, pour sa part, 42 dans sa résidence (dont 11, il est vrai, au grenier) ; Hocquart, en possède 9 ; Bégon a le portrait de son frère, l'évêque de Toul. Quant à Dupuy, il s'est fait portraiturer avec les membres de sa famille, avant de quitter Paris, par le grand peintre Largillière ${ }^{64}$ (qui avait aussi fait le portrait du maréchal d'Estrées ${ }^{65}$ dont nous avons parlé plus haut). Il possède également des sculptures de prix, qu'il avait apportées avec lui au Canada: le buste de sa mère et celui de Louis XIV par Coysevox, dont il était vraisemblablement l'ami. Notons qu'un autre de nos intendants a aussi des sculptures fort intéressantes, Antoine-Denis Raudot: "neuf bronzes représentant différentes figures tant grandes que petites dont une à cheval", prisées 400 livres; à l'item 82 de son inventaire nous lisons: "un bronze sur son pied de bois représentant la Flagellation de Notre Seigneur et deux petites figures chinoises en bois" (90 livres), d'autres bronzes moins dispendieux, et plusieurs figures (de peu de valeur cependant) en pierre de lard. On retrouve d'ailleurs dans l'inventaire de son père, Jacques Raudot, quatre statuettes antiques en bronze et en cuivre doré.

Il y aurait grand intérêt à connaître les sujets des tableaux (comme aussi des tapisseries et des estampes) de nos personnages. Malheureusement les mention.s en sont trop rares pour que l'on puisse en tirer tout le parti désirable. Par exemple sur les 115 tableaux d'Antoine-Denis Raudot, 100 sont simplement énumérés sans titre. Deux cas seulement nous donnent des éléments valables: De Meulles (129) et Talon (98). Voyons la part respective de chacun. Sujet religieux: 24 et 14 ; sujet profane: 83 et 35 ; le roi ou la famille royale: 4 et 4 ; antiquité classique: 3 et 12; sans mention de sujet: 15 et 33 . La part des sujets religieux est loin d'être médiocre, mais notons que pour De Meulles quinze de ces tableaux décorent la chapelle du Château. La fidélité monarchique nous semble l'un des traits caractéristiques de ces administrateurs; nous le constatons ici. La sobre décoration de la chambre de Talon, rue du Bac, comprenait "une estampe représentant le Roy", et une autre "représentant le Roy d'Angleterre", sans doute Jacques II, car il était très lié avec les rilieux jacobites.

Dans presque tous les inventaires nous retrouvons des tapisseries, selon la mode du temps (les deux exceptions sont Bégon et

64 J.-C. Dubé, op. cit., 378.

$65 \mathrm{DBF}$. 
Hocquart) ; on y voit plusieurs noms des grands lieux de fabrication: Bruges, Beauvais, les Gobelins, Parme, Bergame, Venise, l'Auvergne. Quelques-uns en possèdent plusieurs, valant relativement peu; à la Source il y en a dans toutes les pièces, mais la plus précieuse ne passe pas les 420 livres. Par contre d'autres en ont de très riches; dans l'appartement de Louis Robert on peut voir "une tenture de tapisserie contenant six pièces de jeux d'enfants, faisant vingt quatre aulnes et demy de court sur trois aulnes et un douze de hauteur, doublée de plain de toille verte de tapisserie d'Angleterre, prisé sept mil livres". Dupuy avait apporté à.Québec une tapisserie des Flandres en cinq pièces représentant l'Histoire d'Abraham en grands personnages sur des dessins de Jules Romain.

La décoration de leurs résidences comporte aussi bien des éléments dont nous ne retiendrons que les plus importants comme les meubles, les porcelaines ou quelque objet rare ou précieux que l'on garde soigneusement dans un tiroir ou une montre.

Ce qui frappe dans leur mobilier, chez la plupart, c'est la sobriété, mais une sobriété pour laquelle on ne sacrifie jamais le confort et le bon goût; celui de Hocquart est exemplaire à cet égard: les meubles sont solides et en très bon état, le foyer est garni avec beaucoup de goût: quelques beaux bibelots, une pendule signée Gosselin. C'est ce qu'on retrouve ailleurs. Beauharnois a quelques beaux meubles, dont "sept fauteuils de bois doré aux armes de sa famille". De Meulles possède des meubles "de la Chine"; plusieurs fauteuils et chaises sont recouverts de tapisserie, selon la mode du temps, plusieurs porcelaines de Hollande ornent les cheminées. Rien de très dispendieux. Seul Bégon semble plus mal partagé: plusieurs de ses meubles sont vieux et en mauvais état. Vraisemblablement il ne s'était pas remis des importantes pertes d'argent qu'il avait subies en Canada.

Deux intendants tranchent sur les autres cependant par leur luxe: Dupuy et Louis Robert. Le premier s'attendait sans doute de passer de nombreuses années au Canada; il avait apporté à grands frais, entre autres choses, un mobilier important et assez luxueux: des chaises et fauteuils recouverts en damas vert, des bureaux de marquetterie, de nombreuses pièces décoratives, plusieurs caisses de glaces, deux tables de marbre. L'autre, qui avait fait du château de la Fortelle, en Brie, l'un des rendez-vous mondains de la banlieue parisienne ${ }^{66}$, avait un mobilier très

66 E. Mireaux, Une province française au temps du grand Roi. La Brie (Paris, 1958), 264ss. 
luxueux - je ne mentionne qu'une table de marbre jaspé (prisée 250 livres), 18 chaises de bois de noyer sculpté, un cabinet de la Chine (600 livres), plusieurs miroirs de prix (dont un de 550 livres) et une pièce en comportant 45 (prisés 1250 livres), appelée d'ailleurs le "grand cabinet de glace". Il peut montrer à ses visiteurs une "bague d'un gros diamant rozette", évaluée à 2000 livres, et une "boucle de ceinture de femme de 13 diamants rozette de mediocre grosseur" (prisée 800 livres). La chambre à coucher renferme quelque chose de fort beau, mais qu'il est amusant de comparer à ce que comporte celle d'un autre intendant; le lit vaut 3000 livres, il comprend une couche à hauts piliers, richement décorée (velours de Venise, satin couleur de cerise, broderie de soie), six fauteuils de ruelle, un fauteuil de commodité, un lit de repos et quatre chaises. Le reste du mobilier, dont un miroir de 300 livres, est estimé à 422 livres. Voyons maintenant ce que Jean Talon laisse dans sa chambre en 1694: "une couche à haults pilliers de bois de noyer garnie de son enfonçure, sommier de crain, deux matelas remplis de laine, un traversin de coutil remply de plume, une couverture de laine blanche, une autre piquée, le tour dudit lit garny d'une housse de serge d'aumalle verte garnye autour d'une frange fausse, prisé ensemble la somme de 40 livres". Le reste du mobilier et de la décoration est évalué à 75 livres, 5 sols. L'atlas, déjà mentionné, vaut à lui seul 30 livres; le reste bien peu: un bureau de bois de noyer (4 livres), deux chaises de paille ( $10 \mathrm{sols})$, quelques tableaux et estampes de peu de valeur, et un "Christe d'yvoire posé sur sa croix de bois de violette sur un fonds de velours noir garnye de sa bordure dorée" (peut-être 7 livres).

Le contraste est frappant. Si le sieur Robert n'a pas voulu prendre son poste en Canada en 1663, c'est peut-être qu'il craignait de ne pouvoir y retrouver le confort et le luxe de France.

Qu'ils aient sacrifié à la mode ou qu'ils aient eux-mêmes pratiqué les jeux de hasard (c'est sûr pour Louis Robert, qui, semble-t-il, a servi de modèle à La Bruyère pour son portrait du joueur ${ }^{67}$ ), ils ont tous des tables à jouer chez eux. Soit une "table à jouer ou quinola sur quatre pieds garnie d'un tapis de drap vert", comme chez Bégon, qui en a trois autres comme celle-là (le jeu expliquerait-il sa relative pauvreté ?) ; soit un "tore trac d'ébeine garni de ses dames et cornets", chez Louis Robert, ou un "tric trac... et jeu de loto", tels qu'on les voit dans l'appartement de Gilles Hocquart. Raudot possède aussi

67 Ibid., 266. 
un petit billard (prisé 10 livres) et Dupuy "quatre billards et bistoquets a jouer au billard et billes de différentes grosseurs". Le seul à ne posséder aucun meuble de cette espèce est Talon.

Il nous reste un dernier art à mentionner, la musique: mais s'agit-il d'une activité plutôt artistique que sociale ? Nous ne saurions toujours dire. Louis Robert a un grand clavecin (prisé 200 livres); De Meulles, une "epinette avec son sousbassement de bois de noyer" (prisée 125 livres). Quant à Dupuy, il avait apporté avec lui à Québec quelques instruments: "deux basses de viole anglaises avec les etuys et archets"; "une petite epinette portative et un grand cabinet d'orgue, contenant douze jeux, avec son sommier et sa soufflerie". L'on peut penser qu'il s'intéressait activement à ces instruments, puisque sa bibliothèque contenait plusieurs cahiers et livres de musique et que, même après la saisie de ses biens, on le retrouve à Paris avec une basse de viole, mentionnée dans l'inventaire, peu volumineux, de ce qu'il lui restait de biens en 17:38

Cette revue rapide de quelques données d'ordre intellectuel s'avère donc riche de renseignements pour qui veut connaître un peu mieux la personnalité des intendants que le roi destinait au Canada. Rappelons quelques-uns des traits marquants que nous avons dégagés.

Tous sont d'un milieu où les choses de l'esprit sont à l'honneur; il y a des diplômés dans leur parenté, mais aussi de véritables intellectuels, voire des collectionneurs réputés; et parfois il s'agit d'une tradition plus que séculaire. Leur éducation a été soignée, comme il sied dans un milieu de robins. Le cas de ClaudeThomas Dupuy ne fait pas exception; son père qui était un des gros marchands-papetiers de Paris ${ }^{68}$, voulait sans doute parfaire dans son fils qu'il fit étudier, l'ascension sociale de la famille. L'étude de leur mobilier - de leur bibliothèque surtout - nous a révélé des hommes intéressés au droit, aux lettres ou à la religion, et qui n'étaient pas insensibles aux attraits de l'art, peinture, sculpture, voire musique. Nous n'avons certes pas rencontré de grande collection, semblable à celles que F. Bluche a retrouvées chez certains magistrats parisiens; mais par ailleurs ces intendants nous ont paru à ce niveau représentatifs de la masse des robins de la métropole, et donc très intéressants.

Pourtant à certains égards, quelques-uns d'entre eux émergent de la masse. Quatre de ces personnages peuvent être consi-

68 H.-J. Martin, op. cit., 726. 
dérés comme faisant partie de cette République des Lettres, dont nous parlions au début: le numismate Claude de Bouteroue, l'administrateur-savant de la Compagnie des Indes, AntoineDenis Raudot, le physicien Claude-Thomas Dupuy (ces deux derniers possédaient d'ailleurs des bibliothèques remarquables) ${ }^{68 a}$ et François de Beauharnois, le correspondant de Dom Montfaucon. L'appartement de Louis Robert de la Fortelle nous a semblé d'un luxe raffiné, mais sans exagération.

Sur beaucoup de points abordés ici nous n'avons pu donner que quelques indications. Deux raisons à cela: les limites d'un article ne permettent pas une analyse plus poussée, et dans de nombreux cas il a fallu nous contenter de documents imparfaits ou même nous résigner à d'importantes lacunes documentaires.

Il resterait à mesurer le rayonnement intellectuel des administrateurs dans la colonie - question sur laquelle encore peu d'études ont été faites. Mais la documentation pertinente existe-telle? Nous avons vu Talon assister à une soutenance publique au Collège des Jésuites, nous savons que Dupuy était souvent consulté par les Québécois à cause de ses connaissances scientifiques ${ }^{69}$, et que les Raudot donnaient des concerts au Palais ${ }^{70}$ où ils invitaient la belle société de la Capitale. Il y aurait peut-être moyen de trouver des renseignements complémentaires.

Sans doute serait-il possible aussi, et instructif, de faire des comparaisons avec les autres groupes d'administrateurs français de la colonie, les gouverneurs, par exemple, les évêques, les officiers d'armée; ou avec les intendants des provinces françaises. Voire avec les administrateurs des colonies anglaises où Pierre Kalm trouvait, à tort sans doute, que les sciences étaient "tenues dans un mépris universel", tandis qu'en Nouvelle-France les "gens de distinction avaient bien plus de goût pour l'histoire naturelle et les lettres" ${ }^{71}$.

68a Nous espérons, en poursuivant nos recherches sur Bouteroue, trouver des précisions sur ses biens, sa bibliothèque en particulier.

69 J.-C. Dubé, op. cit., 317 .

70 DBC.

71 L. W. Marchand (éd.), Voyages de Kalm en Amérique (Montréal, $1880)$, II $: 6$. 